\title{
Performance Improvement of Physically Based Spectral Rendering Using Stochastic Sampling
}

\author{
Shin Watanabe, Shota Kanamori, Sho Ikeda, \\ Bisser Raytchev, Toru Tamaki, and Kazufumi Kaneda \\ Department of Information Engineering, Graduate School of Engineering, \\ Hiroshima University, Higashi-hiroshima, Japan \\ \{watanabe, kanamori, ikeda\}@eml.hiroshima-u.ac.jp, \\ \{bisser, tamaki,kin\}@hiroshima-u.ac.jp
}

\begin{abstract}
In recent years, many researchers in computer graphics pay attention to spectral rendering where light transport is modeled with multiple wavelengths instead of just using the red, green and blue components. This paper discusses some of the advantages of spectral rendering compared to traditional RGB rendering. For the practical use of spectral rendering, it is important to improve the performance of calculating the spectral distribution of light. The paper also proposes a method for efficiently sampling the wavelength of light in photon mapping-based rendering.
\end{abstract}

Keywords: Spectral rendering, Global illumination, Stochastic sampling, Spectral distribution, Photon mapping.

\section{Introduction}

Display monitors usually use only red, green and blue color components. Based on the mixture of these three components, display monitors provide most of the colors we can perceive. Conventional computer graphics renderers also use a RGB color system in the computation of light transport. That is, the color of light sources and the reflectance of objects are specified in R, G and B components, and the light transport between the light sources and objects is also calculated in the three-color components. The computation of light transport in the RGB color system, however, does not always give good results. Dispersion, diffraction and interference of light are clear examples of the limitations of the RGB rendering. Physically based spectral rendering where light transport is calculated with the spectral distribution of light is a vital approach to overcome these limitations.

Global illumination algorithms are also important in the computation of light transport. These algorithms calculate both direct and indirect light, and generate photo-realistic images. Radiosity methods and Monte-Carlo raytracing algorithms are common global illumination algorithms. The photon mapping algorithm [1] is classified into the group of Monte-Carlo raytracing algorithms,

S. Tominaga, R. Schettini, and A. Trémeau (Eds.): CCIW 2013, LNCS 7786, pp. 184-198, 2013.

(C) Springer-Verlag Berlin Heidelberg 2013 
and widely used because of of its capability to render specular reflections such as caustics. It is not so difficult to expand the photon mapping algorithm to be able to handle the spectral distribution of light. However, a straightforward extension brings a huge computational cost.

Traditional computer graphics has not paid much attention to a sampling methods for the wavelength of light. Most of the rendering methods that pursue a photo-realism just care about the number and the direction of sampled ray in $3 \mathrm{D}$ space. It is an important subject to investigate the ray sampling in the spatial domain, but a sampling strategy in the spectral domain is also important in physically based rendering.

In this paper, we first discuss the limitations of conventional RGB rendering methods and the usefulness of the physical based spectral rendering, demonstrating rendered images by a photon mapping with spectral distribution of light. We also propose a method for accelerating the photon mapping with spectral distribution by introducing an importance sampling of wavelengths of light in a photon tracing process.

\section{$2 \quad$ RGB Rendering vs. Spectral Rendering}

It is important to investigate the color system in the calculation of light transport. Visible light is electro-magnetic waves and we perceive different colors depending on the wavelength. Mixture of colors also generates a different color. Making use of the property of the color mixing system, color monitors can display a huge amount of colors using only a few color components.

As is well known, our eye has three types of cone cells: L, M and S, and the stimulus ratio of these cone cells determines the perceived colors. The wavelengths to which the $\mathrm{L}, \mathrm{M}$ and $\mathrm{S}$ cone cells are most sensitive are almost equal to those of the R, G, and B color components, respectively. Therefore, color display monitors usually use these three primary colors.

The R, G and B color components are also usually used in the calculation of light transport in computer graphics because of the background mentioned above. The RGB rendering works well in many cases, but it is not a physically based rendering, because the interaction of light and objects depends on the wavelength of visible light, not RGB components. Although we don't recognize spectral distributions directly (see Fig. 1), the spectral distribution of light should be taken into account in the computation of light transport.

\subsection{Images Rendered by RGB Rendering and Spectral Rendering}

Most of the conventional rendering methods use the RGB color model, where the R, G and B color components of the light sources and the reflectance of the objects are specified, and the light transport from the light sources to the objects and finally to a viewpoint is computed using the R, G and B components. However, this RGB rendering sometimes gives quite incorrect results. Figure 2 shows a dispersion phenomenon rendered by RGB rendering and physically based 


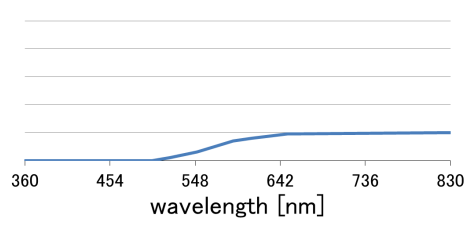

(a) Spectral distribution $\mathrm{A}$

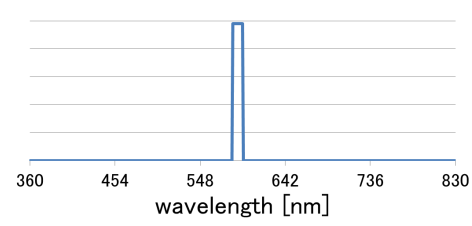

(b) Spectral distribution B

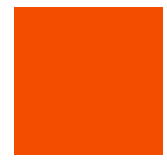

(c) Color

Fig. 1. Different spectral distributions giving the same color perception.

Note that we can perceive the same color from different spectral distributions. This is because the stimulus ratio of the three cone cells is same.
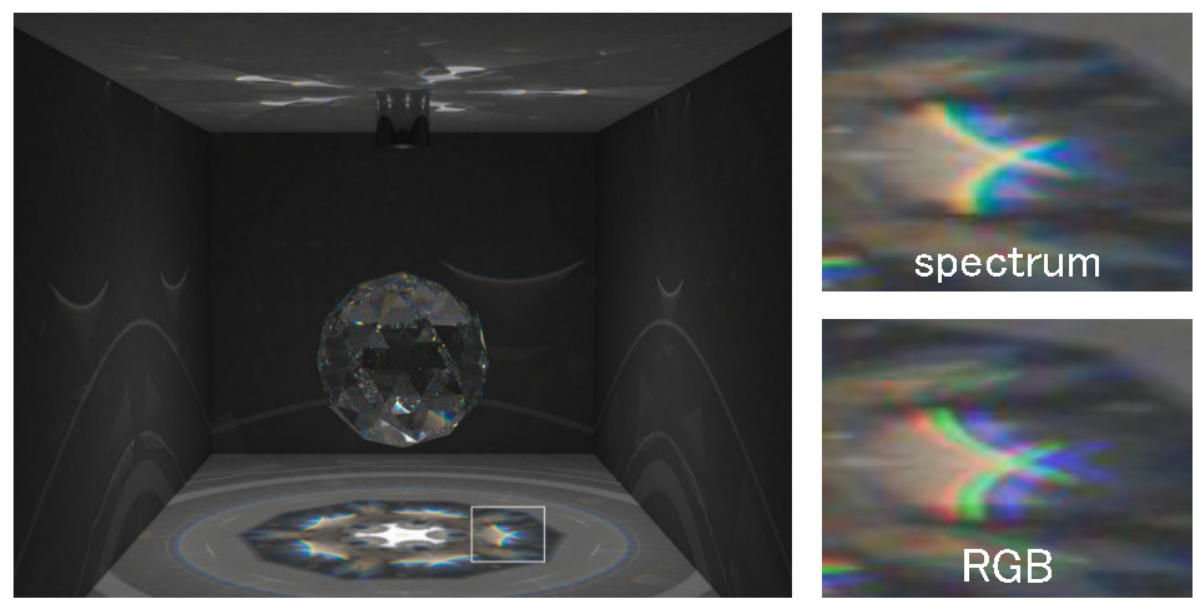

Fig. 2. Dispersion of light

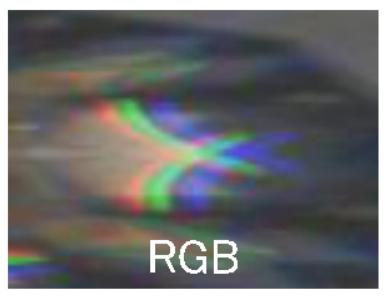

spectral rendering. The RGB rendering generates an erroneous result, since the direction of refracted light greatly depends on the wavelength of light, and the RGB rendering cannot sample a sufficiently wide range of wavelengths with only three components. RGB rendering also cannot simulate diffraction effects and phenomena related to the interference of light.

Even in the case of a diffuse reflection environment, the RGB rendering and the spectral rendering generate different images. Figure 3 compares two images generated by RGB rendering and spectral rendering. The spectral reflectance of objects is specified in the spectral rendering, while the reflectance of the $R, G$ and $\mathrm{B}$ components with the same color as the spectral reflectance is provided in the RGB rendering. For example, the spectral reflectance of a sphere and the converted reflectance of R, G and B components are shown in Fig. 4 (c) and (d), respectively. The spectral distribution of the light source is set to uniform in Fig. 3 (a), while a sodium lamp with a bright line spectrum is used in Fig. 3 (b). The spectral distribution of the sodium lamp and the converted RGB components are shown in Fig. 4 (a) and (b), respectively. 
In the case of the light source with uniform spectral distribution (Fig. 3 (a)), the RGB rendering and the spectral rendering generate almost the same images, while in the case of the sodium lamp (Fig. 3 (b)), the color of the sphere in the RGB rendering is quite different from that of the spectral rendering. The color of the sphere is black in the actual scene, because the spectral reflectance of the sphere is zero at the corresponding wavelength in the bright line spectrum of the sodium lamp. On the other hand, in the RGB rendering, the converted RGB components of the sphere's reflectance (Fig. 4 (d)) are multiplied by the converted RGB components of the light source (Fig. 4 (b)) to compute the light transport. This results in the erroneous color of the sphere (the left side of Fig. $3(\mathrm{~b}))$.

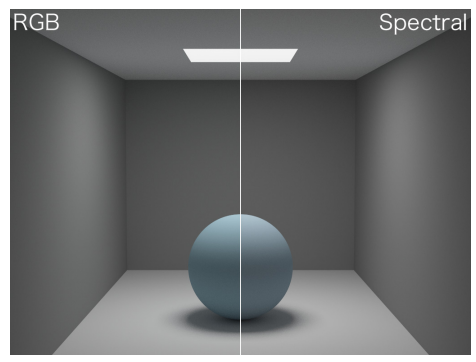

(a) Light source with uniform spectral distribution

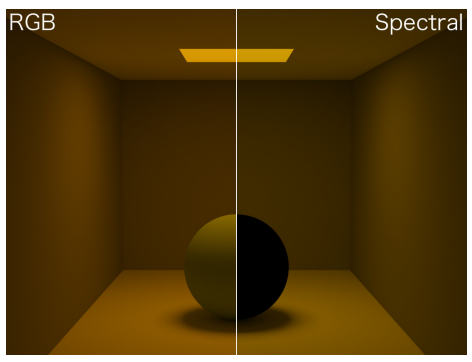

(b) Sodium lamp with bright line spectrum

Fig. 3. Comparisons of RGB and Spectral renderings

Although the previous example is an extreme case, the RGB rendering has a chance to generate erroneous results in many cases. Figure 5 shows an example where a Stanford dragon is lit by a fluorescent lamp. The spectral distribution of the fluorescent lamp and the spectral reflectance of the Stanford dragon are shown in Fig. 6. As shown in four regions of Fig. 5, the RGB rendering cannot generate an accurate image, even though the differences of the rendered images are subtle.

\subsection{Theoretical Considerations of RGB and Spectral Renderings}

As demonstrated in the previous section, the RGB rendering has limitation on the accuracy of rendering. The RGB rendering generates a plausible result only when an object is lit by a light source with uniform spectraldistribution under diffuse reflection environment. Let us consider theoretically the reason why the RGB rendering cannot generate accurate images.

The calculation process for reflected intensity of light is shown in Fig. 7. In the real world, an emitted light from a light source has a spectral distribution $I(\lambda)$. The reflectance of an object changes depending on the wavelength of light, i.e., the reflectance is a function of the wavelength, and we can express it as a 


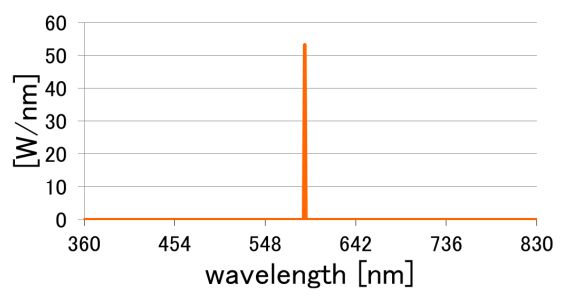

(a) Spectral distribution of the sodium lamp

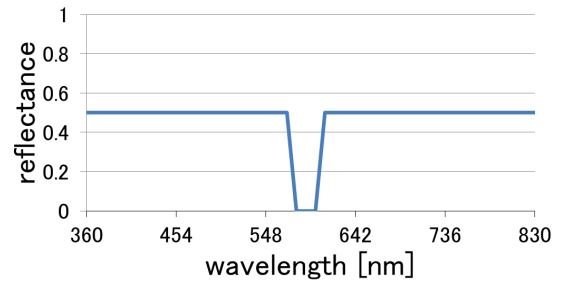

(c) Spectral reflectance of the sphere

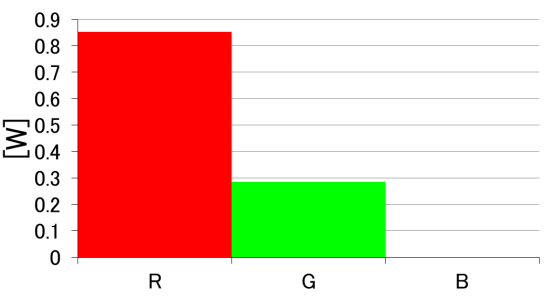

(b) Converted RGB color components

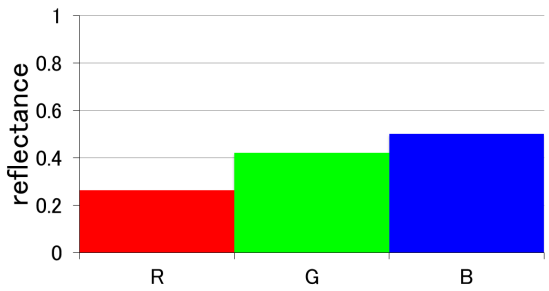

(d) Converted RGB reflectance

Fig. 4. The spectral distribution of the light source and the spectral reflectance of the sphere in Fig. 3

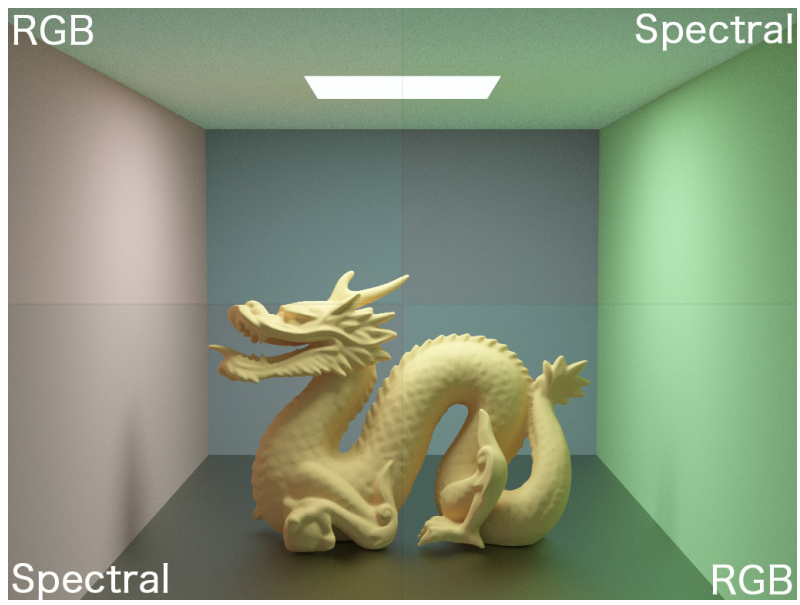

Fig. 5. The Stanford dragon lit by a fluorescent lamp 


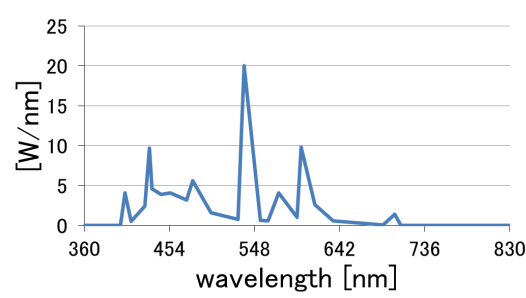

(a) Spectral distribution of a fluorescent lamp

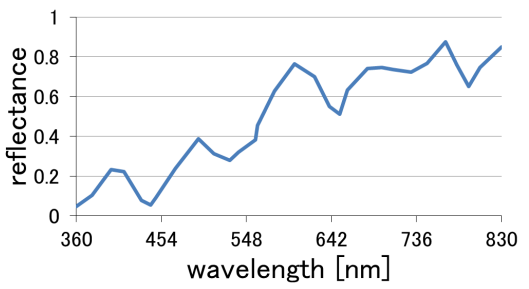

(b) Spectral reflectance of a Stanford dragon

Fig. 6. The spectral distribution of a light source and the spectral reflectance of an object in Fig. 5

spectral reflectance $\rho(\lambda)$. The reflected light from an object can be expressed by the product of $\rho(\lambda)$ and $I(\lambda)$. The reflected light also has a spectral distribution. Spectral rendering faithfully simulates this process, and finally the spectral distribution of the reflected light is converted into RGB components for display on a RGB monitor, taking into account the color matching functions (see Fig. 8).

On the other hand, in the RGB rendering, the spectral distribution of emitted light and the spectral reflectance are first converted into RGB components $\left(I_{R}, I_{G}, I_{B}\right)$ and $\left(\rho_{R}, \rho_{G}, \rho_{B}\right)$, respectively. Then, the reflected light is calculated by multiplying each component of $\left(I_{R}, I_{G}, I_{B}\right)$ and $\left(\rho_{R}, \rho_{G}, \rho_{B}\right)$. The reflected light is expressed as RGB components, and is directly displayed on a RGB monitor. However, the resultant RGB components are different from the actual ones because the conversion from spectral to RGB components is not a linear operator.

\section{Related Work}

In spectral rendering, there are several ways to sample a spectral distribution. A uniform sampling over the bandwidth of visible light is the easiest way to realize a spectral rendering. It is not so difficult to extend a conventional RGB renderer to a spectral renderer introducing the uniform sampling, and several renderers employ this strategy. The computational cost, however, increases proportionally to the number of sampled wavelengths. For example, if we sample the spectral distribution of visible light at $10 \mathrm{~nm}$ interval, the computational cost will be more than 10 times larger than that of the RGB rendering.

A spectral distribution can be transformed by using basis functions. This approach is an alternative way to realize a spectral rendering. When a spectral distribution is smooth enough, i.e., the spectral distribution does not have high frequencies, the transformed spectral distribution can reduce the computational cost. However, the spectral distributions of artificial light sources, such as fluorescent lamps, often contain bright line spectra. This results in a high computational cost. To address the problem, a composite spectral model [2] was 


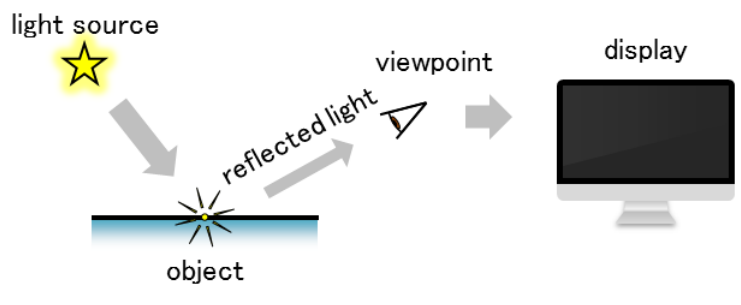

\begin{tabular}{lccc}
\hline $\begin{array}{c}\text { Physically based } \\
\text { Spectral rendering }\end{array}$ & RGB rendering \\
spectrum of light source & $I(\lambda)$ & II & $I_{R}, I_{G}, I_{B}$ \\
reflectance of object & $\rho(\lambda)$ & II & $\rho_{R}, \rho_{G}, \rho_{B}$ \\
reflected light & $\rho(\lambda) I(\lambda)$ & & $\rho_{R} I_{R}, \rho_{G} I_{G}, \rho_{B} I_{B}$ \\
& $R, G, B$ & & directly \\
display & & & $R^{\prime}, G^{\prime}, B^{\prime}$
\end{tabular}

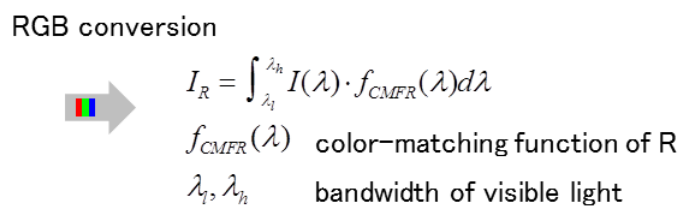

Fig. 7. The rendering process for RGB and Spectral renderings

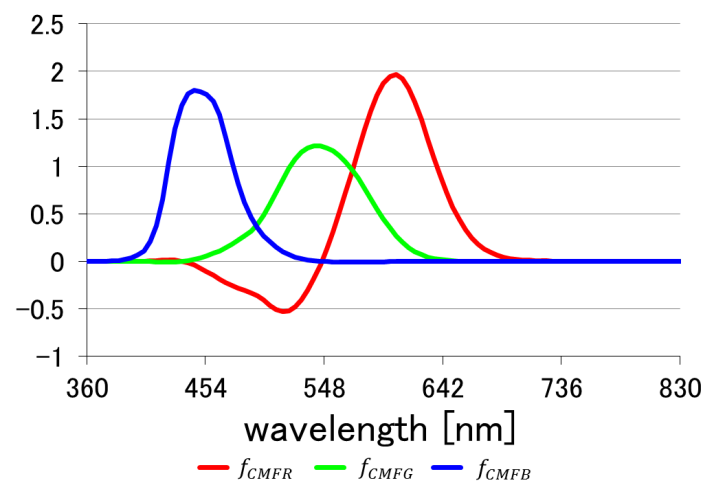

Fig. 8. Color-matching functions 
proposed, where a spectral distribution is approximated with a low degree of basis functions, and bright line spectra are separately expressed by using delta functions.

The latest research on a photorealistic rendering mainly focuses on ray sampling in the spatial domain. For example, Knaus, et al. [7] proposed a formulation of progressive photon mapping based on a probabilistic approach, and successfully rendered translucent objects with a constant amount of memory. It is an important subject for rendering to estimate the radiance in a scene with a limited number of photons. To solve this problem, many researchers have investigated sampling strategies of shooting direction of photons in a $3 \mathrm{D}$ space. In this paper, we focus on a method for sampling the wavelength of light, which is an important subject in physically based photorealistic rendering.

Turning to global illumination algorithms, photon mapping (PM) [1] is a suitable algorithm for implementing spectral rendering. The algorithm consists of two passes of ray tracing (see Fig. 9). Photons emitted from a light source are traced in the first pass, and the photons that collide with non-specular surfaces are recorded in a photon map. In the second pass, viewing rays are shot from a viewpoint, and the intensity of light is calculated by using a photon map on the surface where the viewing ray intersects. To calculate an accurate intensity of light, the algorithm requires a huge number of photons.

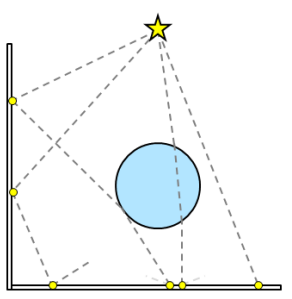

1. Photon tracing \& Photon map creation
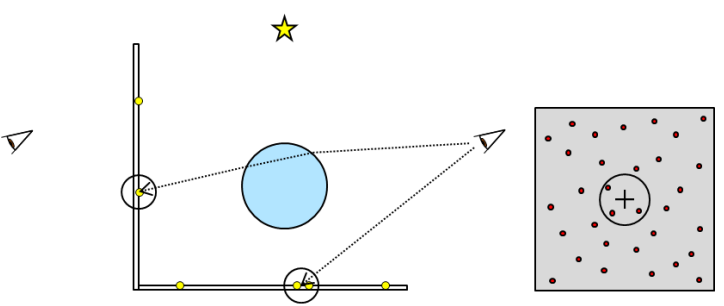

2. Ray tracing \& Photon density estimation

Fig. 9. Photon mapping

Progressive photon mapping (PPM) [3] solves the above problem of PM. In contrast to PM, PPM first calculates intersections with viewing rays and surfaces, and then the emitted photons from the light sources are traced. PPM has an additional advantage; Photon maps are updated in conjunction with the photon tracing pass, and the memory size required to store the photon maps is lower than that of PM. This is a good property for spectral rendering, because spectral rendering requires a larger memory to store the spectral distribution of light.

Several methods were developed to implement spectral rendering by PM. Gutierrez, et al. [4] proposed a single wavelength photon model, where each photon has a different single wavelength. They rendered fluorescent materials 
taking into account dispersion of light. The single wavelength photon model can trace photon paths exactly based on physical laws, but a number of photons are required to represent a spectral distribution.

On the other hand, Boudet, et al. [5] and Lai, et al. [6] proposed a multiwavelength photon model, where a photon has multiple wavelengths sampled over the bandwidth of visible light. The multi-wavelength photon model can generate an image with a smaller number of photons. However, the multi-wavelength photon model cannot render wavelength-dependent phenomena such as dispersion, diffraction and so on. To address this problem, Lai, et al. [6] employed a method combining a single wavelength photon and a multi-wavelength photon models.

\section{Performance Improvement of Spectral Photon Mapping}

We propose a method for accelerating photon mapping based on a single wavelength photon model and a multi-wavelength photon model. The proposed method stochastically samples the wavelength of visible light.

\subsection{A Single Wavelength Photon Model}

In the cases of dispersion, refraction and interference of light, we use the single wavelength photon model where each photon has a single wavelength. In our spectral renderer, single wavelength photons are emitted from the light source, and the wavelength of each photon is determined by a probability distribution function based on the spectral distribution of the light source. The emission probability $p(\lambda)$ of a photon with wavelength $\lambda$ is determined by the following equation:

$$
p(\lambda)=\frac{I(\lambda)}{\int_{\lambda_{l}}^{\lambda_{h}} I(\lambda) d \lambda},
$$

where $I(\lambda)$ is the spectral distribution of the light source, and $\left[\lambda_{l}, \lambda_{h}\right]$ designates the bandwidth of visible light. Our sampling strategy is based on importance sampling, and reduces the number of photons in comparison with random sampling.

\subsection{A Multi-Wavelength Photon Model}

In the multi-wavelength photon model, a single photon has multiple wavelengths over the bandwidth of visible light, and this model is usually used for diffuse reflection environment. It is important to control the number of photons in the photon tracing pass. If we generate reflected photons always when an incident photon collides with a diffuse surface, a huge number of photons with small radiant power would be generated, and this would result in a high computational cost. We should quit the photon trace taking into account both the reflectance of the surface and the radiant power of the photon. 
The traditional photon tracing method used in PMs and PPMs determines the continuation of the photon trace taking into account only the reflectance of a surface. In our proposed method, it is determined by taking into account both the reflectance of the surface and the radiant power of the photon.

Let's assume the radiant power of an incident photon is $\boldsymbol{I}$, the radiant power of the light source originating the incident photon is $\boldsymbol{I}_{L}$, and the spectral reflectance of a surface is $\boldsymbol{\rho}$. The relative radiant power of the incident photon is defined by $\boldsymbol{I}_{r}=\boldsymbol{I} / \boldsymbol{I}_{L}$, where the division symbol means a component-wise division. The continuation of photon trace is determined by using the following probability:

$$
p=\max _{1 \leq i \leq N}\left(\boldsymbol{\rho} \odot \boldsymbol{I}_{r}\right)_{i}=\max \left(\rho_{1} I_{r 1}, \rho_{2} I_{r 2}, \cdots, \rho_{N} I_{r N}\right),
$$

where $N$ is the number of sampled wavelengths, and the symbol $\odot$ means a component-wise multiplication. Note that we use the relative radiant power to determine the continuation of a photon trace. The relative radiant power also contains the tracing history of the photon. If the relative radiant power has a larger value, the rendering scene may contain more surfaces whose spectral reflectance contribute to the radiant power of the incident photon. This strategy can decide the continuation of photon trace more efficiently.

A reflected photon is generated probabilistically based on the probability $p$ as shown in Eq. 2. Based on the theory of Monte-Carlo sampling, the radiant power of a reflected photon is $\boldsymbol{I}_{0}=\boldsymbol{\rho} \odot \boldsymbol{I} / p$. Using the proposed method, photons having a larger contribution to the rendering scene have higher possibility to survive.

\section{Results}

\subsection{Single Wavelength Photon}

The proposed method for the single wavelength photon model is examined under a non-diffuse environment where a transparent and a specular spheres are located in a box. A point light source with the spectral distribution shown in Fig. 10 (b) is located on the right wall. Figure 10 (a) shows an image rendered by PPM with sufficient number of photons (the viewing ray tracing pass and the photon tracing pass are iterated 18,000 times). The refracted light from the transparent sphere makes caustics on the floor and the specular sphere reflects the surrounding area.

Figure 11 shows the relation between computation time and the root mean square (RMS) errors. We used the image in Fig. 10 (a) as a ground truth. In Fig. 11, a solid line shows the computation time of the proposed method based on importance sampling, while the dashed line is for the case of random sampling. The computational cost was being reduced by about $9 \%$ in the proposed method.

\subsection{Multi-wavelength Photon}

To evaluate the performance of the proposed method in various conditions, we tested the proposed method in three different types of datasets: non-diffuse, 


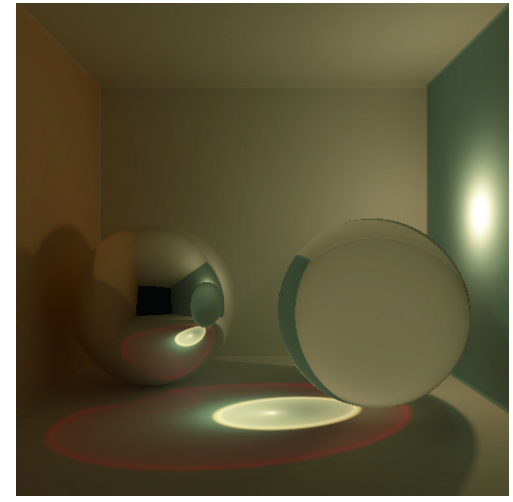

(a) Test scene

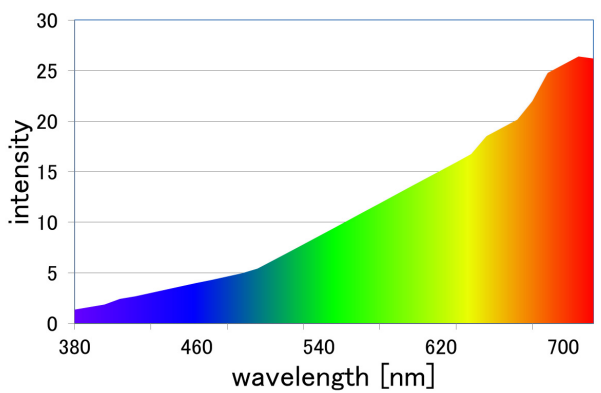

(b) Spectral distribution of a light source

Fig. 10. Single wavelength photon model

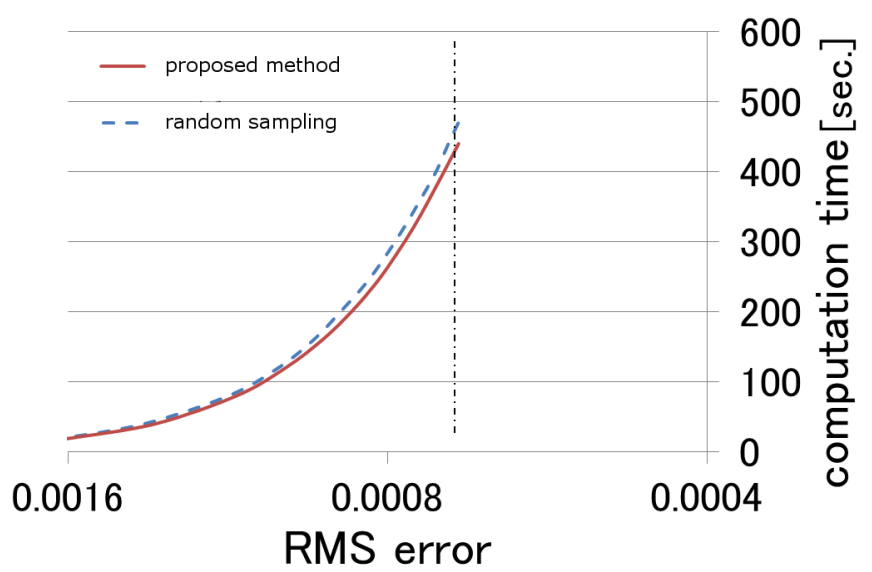

Fig. 11. Computation time of a single wavelength photon model

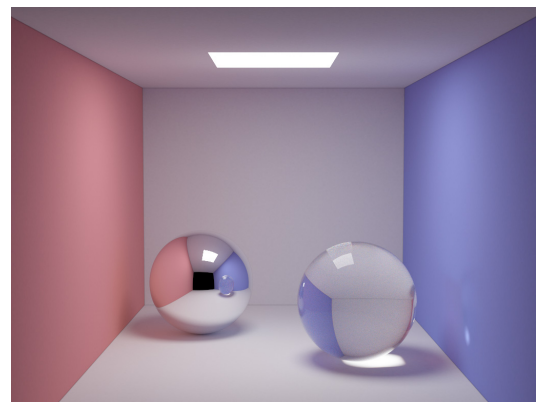

Fig. 12. Dataset of non-diffuse objects 
Table 1. Computation time (second) and reduction rate (\%) for Fig. 12

\begin{tabular}{|c|c|c|c|}
\hline RMS error & Time (Proposed Method) & Time (Traditional Method) & Reduction rate \\
\hline \hline 8.0 & 1644.3 & 1935.5 & 15.0 \\
6.0 & 2658.0 & 3157.5 & 15.8 \\
5.0 & 3672.4 & 4371.4 & 16.0 \\
4.0 & 5839.6 & 6768.5 & 13.7 \\
3.0 & 11203.7 & 12992.2 & 13.8 \\
\hline
\end{tabular}

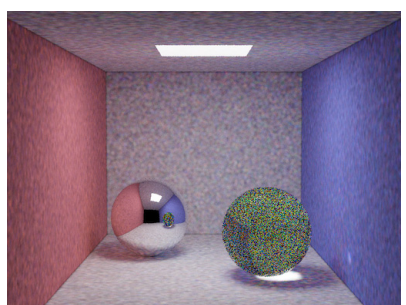

(a) 1 pass RMS error: 31.6

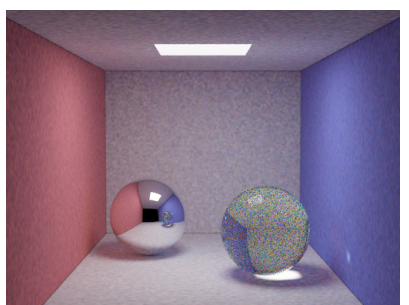

(c) 4 passes RMS error: 21.1

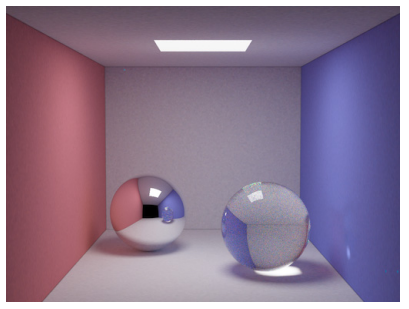

(e) 64 passes RMS error: 5.5

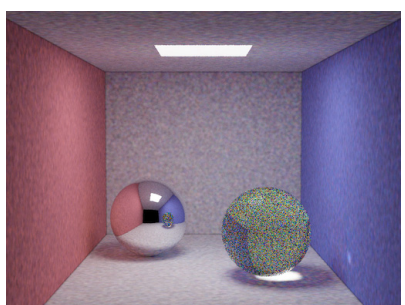

(b) 2 passes RMS error: 26.7

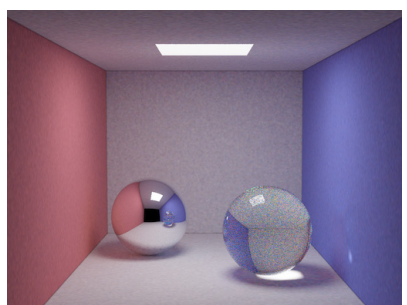

(d) 16 passes

RMS error: 11.0

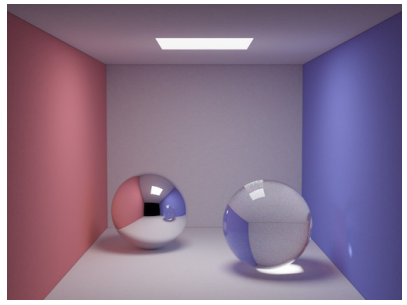

(f) 256 passes

RMS error: 3.3

Fig. 13. Images rendered by the proposed method in increasing number of rendering passes

diffuse, and specular objects. Figure 12 shows a dataset of non-diffuse objects, where a transparent sphere has a constant refractive index over the bandwidth of visible light, i.e., no dispersion of light occurs in the scene. Figure 13 shows images rendered by the proposed method in each rendering pass. RMS errors 
decrease as the number of rendering passes increases. Table 1 and Figure 14 show the computation time in respect to the RMS errors. As in the previous experiment, we again use an image rendered with a sufficient number of photons as a ground truth image. The solid line shows the computation time of the proposed method where the probability of the photon trace is determined by using both the reflectance of a surface and the relative radiant power of a photon, while the dashed line shows a traditional method using only the reflectance of a surface.

Figure 15 shows a dataset of a diffuse object. The computation time is shown in Table 2 and Fig. 16. Finally, Fig. 17 shows a dataset of a specular object, and the computation time is shown in Table 3 and Fig. 18. For the proposed method, the computational costs were reduced by about $15 \%, 21 \%$ and $29 \%$ in the cases of Figs. 12, 15 and 17, respectively.

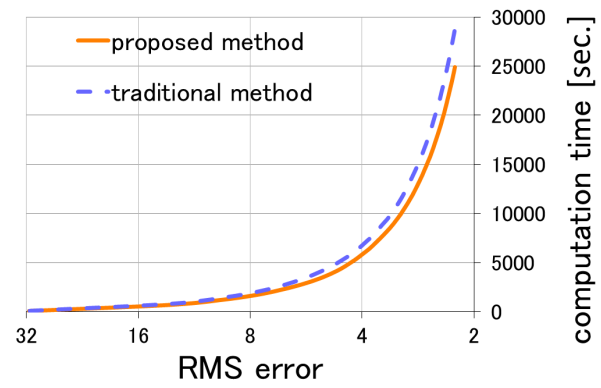

Fig. 14. Computation time for Fig. 12

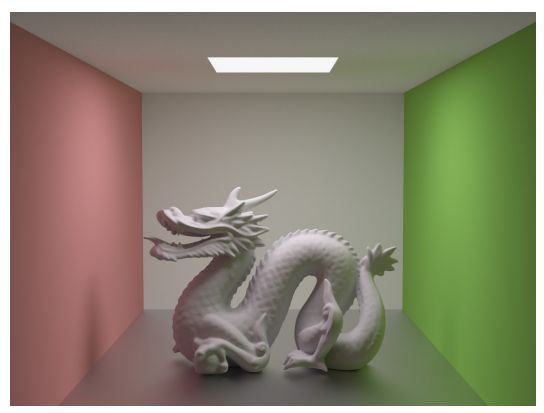

Fig. 15. Dataset of a diffuse object

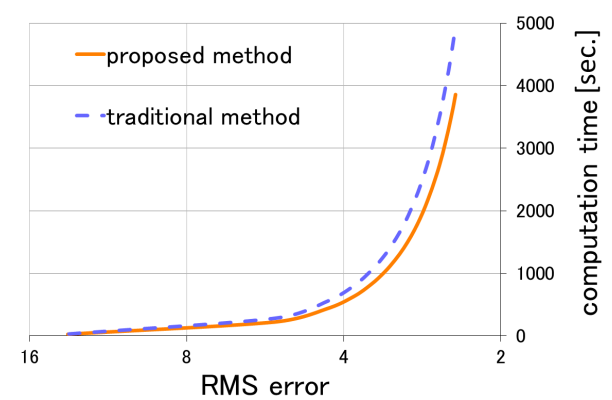

Fig. 16. Computation time for Fig. 15 
Table 2. Computation time (second) and reduction rate (\%) for Fig. 15

\begin{tabular}{|c|c|c|c|}
\hline RMS error & Time (Proposed Method) & Time (Traditional Method) & Reduction rate \\
\hline \hline 6.0 & 210.0 & 266.8 & 21.3 \\
5.0 & 303.5 & 382.1 & 20.6 \\
4.0 & 561.4 & 713.8 & 21.3 \\
3.0 & 1543.8 & 1959.9 & 21.2 \\
2.5 & 3441.4 & 4413.5 & 22.0 \\
\hline
\end{tabular}

Table 3. Computation time (second) and reduction rate (\%) for Fig. 17

\begin{tabular}{|c|c|c|c|}
\hline RMS error & Time (Proposed Method) & Time (Traditional Method) & Reduction rate \\
\hline \hline 30.0 & 279.3 & 396.2 & 29.5 \\
15.0 & 1444.0 & 2044.8 & 29.4 \\
10.0 & 3194.1 & 4517.1 & 29.3 \\
\hline
\end{tabular}

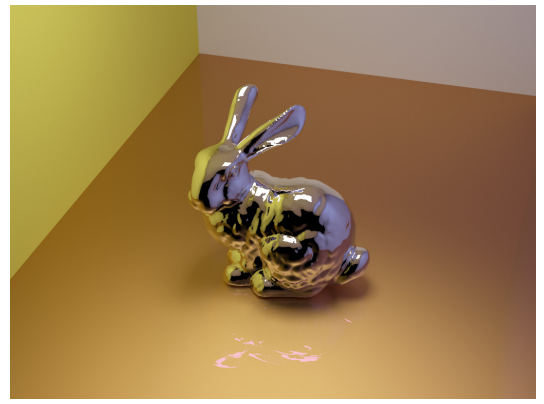

Fig. 17. Dataset of a specular object

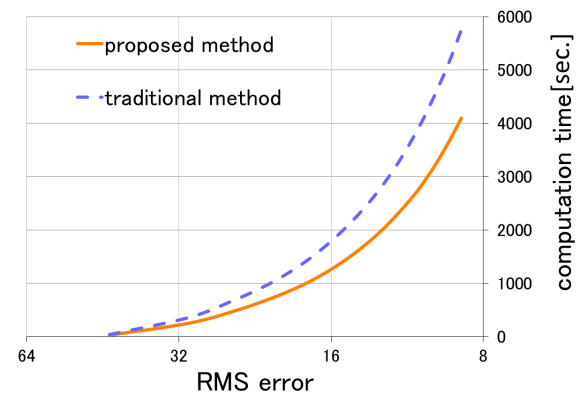

Fig. 18. Computation time for Fig. 17

\section{Conclusions}

In this paper, we investigated the advantages of spectral rendering, and demonstrated that spectral rendering accompanying a global illumination algorithm is a method that can generate physically correct results. We also proposed a method for accelerating spectral rendering by using stochastic sampling techniques for two approaches: single wavelength photon and multi-wavelength photon models. Each approach has advantages in non-diffuse and diffuse environments, respectively. It can be possible to employ both approaches simultaneously in non-diffuse environment to achieve further acceleration, but a futher study on this topic is needed.

Physically based spectral rendering plays an increasingly important role, as the lighting environment in our everyday life changes drastically. In these days, artificial light sources with bright line spectra become more and more popular, such as fluorescent lamps, light emitting diode (LED) lamps, and so on. How to 
sample spectral distributions and spectral reflectance is an important issue in advanced spectral rendering. A further acceleration of physically based spectral rendering is also an important subject.

\section{References}

1. Jensen, H.W.: Global illumination using photon maps. In: Proc. Eurographics Workshop on Rendering 1996, pp. 21-30 (1996)

2. Sun, Y., Fracchia, D.F., Drew, M.S., Calvert, T.W.: A spectrally based framework for realistic image synthesis. The Visual Computer 17(7), 429-444 (2001)

3. Hachisuka, T., Ogaki, S., Jensen, H.W.: Progressive photon mapping. ACM Transactions on Graphics (SIGGRAPH Asia Proceedings) 27(5), Article 130 (2008)

4. Gutierrez, D., Munoz, A., Seron, F.: Non-linear Volume Photon Mapping. In: Proc. Eurographics Symposium on Rendering 2005, pp. 291-300 (2005)

5. Boudet, A., Paulin, M., Pitot, P., Pratmarty, D.: Low Memory Spectral Photon Mapping. In: Proc. WSCG (2004)

6. Lai, G., Christensen, N.J.: A compression method for spectral photon map rendering. In: Proc. WSCG 2007, pp. 95-102 (2007)

7. Knaus, C., Zwicker, M.: Progressive Photon Mapping: A Probabilistic Approach. ACM Transactions on Graphics 30(3), Article 25 (2011) 